\title{
The biomechanical effect of single-level laminectomy and posterior instrumentation on spinal stability in degenerative lumbar scoliosis: a human cadaveric study
}

\author{
Christine M. E. Rustenburg, MD, ${ }^{1}$ Sayf S. A. Faraj, MD, ${ }^{2}$ Roderick M. Holewijn, MD, ${ }^{3}$ \\ Idsart Kingma, PhD, ${ }^{4}$ Barend J. van Royen, MD, PhD, ${ }^{1}$ Agnita Stadhouder, MD, ${ }^{1}$ and \\ Kaj S. Emanuel, PhD'1,5
}

${ }^{1}$ Amsterdam Movement Sciences, Department of Orthopaedic Surgery, Amsterdam UMC, Amsterdam; ${ }^{2}$ Department of Orthopedic Surgery, Radboud UMC, Nijmegen; ${ }^{3}$ Department of Radiology, OLVG, Amsterdam; ${ }^{4}$ Amsterdam Movement Sciences, Faculty of Movement Sciences, Vrije Universiteit, Amsterdam; and ${ }^{5}$ Department of Orthopaedic Surgery, Maastricht UMC+, Maastricht, The Netherlands

OBJECTIVE Degenerative lumbar scoliosis, or de novo degenerative lumbar scoliosis, can result in spinal canal stenosis, which is often accompanied by disabling symptoms. When surgically treated, a single-level laminectomy is performed and short-segment posterior instrumentation is placed to restore stability. However, the effects of laminectomy on spinal stability and the necessity of placing posterior instrumentation are unknown. Therefore, the aim of this study was to assess the stability of lumbar spines with degenerative scoliosis, characterized by the range of motion (ROM) and neutral zone (NZ) stiffness, after laminectomy and placement of posterior instrumentation.

METHODS Ten lumbar cadaveric spines (T12-L5) with a Cobb angle $\geq 10^{\circ}$ and an apex on L3 were included. Three loading cycles were applied per direction, from $-4 \mathrm{Nm}$ to $4 \mathrm{Nm}$ in flexion/extension (FE), lateral bending (LB), and axial rotation (AR). Biomechanical evaluation was performed on the native spines and after subsequent L3 laminectomy and the placement of posterior L2-4 titanium rods and pedicle screws. Nonparametric and parametric tests were used to analyze the effects of laminectomy and posterior instrumentation on NZ stiffness and ROM, respectively, both on an individual segment's motion and on the entire spine section. Spearman's rank correlation coefficient was used to study the correlation between disc degeneration and spinal stability.

RESULTS The laminectomy increased ROM by $9.5 \%$ in FE $(p=0.04)$ and $4.6 \%$ in LB $(p=0.01)$. For NZ stiffness, the laminectomy produced no significant effects. Posterior instrumentation resulted in a decrease in ROM in all loading directions $(-22.2 \%,-24.4 \%$, and $-17.6 \%$ for $F E, L B$, and AR, respectively; all $p<0.05)$ and an increase in NZ stiffness $(+44.7 \%,+51.7 \%$, and $+35.2 \%$ for FE, LB, and AR, respectively; all $p<0.05)$. The same changes were seen in the individual segments around the apex, while the adjacent, untreated segments were mostly unaffected. Intervertebral disc degeneration was found to be positively correlated to decreased ROM and increased NZ stiffness.

CONCLUSIONS Laminectomy in lumbar spines with degenerative scoliosis did not result in severe spinal instability, whereas posterior instrumentation resulted in a rigid construct. Also, prior to surgery, the spines already had lower ROM and higher NZ stiffness in comparison to values shown in earlier studies on nonscoliotic spines of the same age. Hence, the authors question the clinical need for posterior instrumentation to avoid instability.

https://thejns.org/doi/abs/10.3171/2019.2.FOCUS1911

KEYWORDS de novo degenerative lumbar scoliosis; spinal stability; biomechanics; lumbar spinal stenosis

A DULT scoliosis is a 3D deformity of the lumbar spine and is defined by a deviation in the coronal plane of a greater than $10^{\circ} \mathrm{Cobb}$ angle. ${ }^{1}$ Adult patients with scoliosis can be divided into two general groups: those with adult idiopathic scoliosis, originating during child- hood, and those with de novo degenerative lumbar scoliosis, which develops de novo in mostly the 6th decade of life as a result of degenerative processes in the lower back, such as lumbar disc degeneration (DD). ${ }^{1,44,45,52}$ While adult idiopathic scoliosis can lead to Cobb angles greater

ABBREVIATIONS AR = axial rotation; $\mathrm{DD}=$ disc degeneration; $\mathrm{FE}=$ = flexion/extension; $\mathrm{LB}=$ lateral bending; $\mathrm{NZ}=$ neutral zone; $\mathrm{ROM}=$ range of $\mathrm{motion}$.

SUBMITTED January 2, 2019. ACCEPTED February 6, 2019.

INCLUDE WHEN CITING DOI: 10.3171/2019.2.FOCUS1911. 
than $50^{\circ}$, Cobb angles in lumbar spines with degenerative scoliosis mostly do not exceed $40^{\circ} .129,33,45$ Nonetheless, patients with degenerative scoliosis can proceed to develop more disabling conditions, such as spondylolisthesis or spinal canal stenosis resulting in symptomatic neurological compression. . $^{13,45}$

Patients suffering from degenerative lumbar scoliosis and symptomatic spinal canal stenosis often present with low-back pain, radiculopathy, and neurogenic claudication..$^{13,29,30,43}$ When surgery is indicated, a single-level, facet-sparing laminectomy is the most commonly performed treatment to decompress the spinal canal and associated nerve roots. ${ }^{46}$ Subsequently, additional posterior instrumentation composed of pedicle screws and rods can be performed to prevent postoperative instability following laminectomy. However, there is extensive discussion in the literature as to whether posterior instrumentation should be performed to stabilize the spine after the laminectomy. ${ }^{20,35}$ The rationale behind instrumentation is that removal of the lamina is destructive to the anatomy of the lumbar spine and has been shown to alter its biomechanics. ${ }^{3,7}$ Moreover, single-level laminectomy in degenerative lumbar scoliosis might result in progression of the curve or progressive symptomatic postoperative instability of the scoliotic lumbar spine, with an incidence of post-laminectomy iatrogenic spondylolisthesis of $8 \%-31 \%{ }^{8,26} \mathrm{How}-$ ever, previous investigations have focused on patients with spinal stenosis, or they were performed using cadaveric spines without degenerative lumbar scoliosis. . $^{3,6,8,26,48}$ The effects of the facet-sparing laminectomy and the short instrumentation on the stability of the degenerative scoliotic lumbar spine have never been quantified.

Therefore, the first aim of this study was to assess the effects of laminectomy and posterior instrumentation on the stability of lumbar spines with degenerative scoliosis. Spinal stability was analyzed by measuring the range of motion (ROM) and stiffness around the neutral zone (NZ) of the spinal segments. Since intervertebral DD is believed to have a detrimental effect on the ROM and NZ stiffness of the affected spinal segments, ${ }^{41}$ our second aim was to assess the ROM and NZ stiffness of the lumbar spinal segments as a function of the degree of intervertebral DD.

We hypothesized that laminectomy increases the ROM and decreases the NZ stiffness of the treated spinal segments, with only minor or no changes in the adjacent, untreated segments. On the other hand, we hypothesized that there would be a decrease in ROM and an increase in the NZ stiffness of the treated levels following the placement of posterior instrumentation.

\section{Methods \\ Patients}

Fresh-frozen $\left(-20^{\circ} \mathrm{C}\right)$ human cadaveric spines from a population of older donors (mean age $82 \pm 11$ years at time of death) were screened for scoliosis using plain anteroposterior and lateral radiographs (Digital Vet DX-6, Sedecal) to measure the Cobb angle. ${ }^{16,17}$ Ten lumbar spines (T12-L5) with a Cobb angle of $\geq 10^{\circ}$ and the apex on L3 were harvested (range $11.1^{\circ}-41.3^{\circ}$ ). Standard T2-weighted MR images (syngo MR A30, Siemens Symphony 1.5 T; software NUMARIS/4) of the intact lumbar spines were acquired to assess the grade of DD of T12-L1, L1-2, L2-3, L3-4, and L4-5 according to the Pfirrmann classification of T2-weighted midsagittal sections. ${ }^{42}$ Assessment was performed by an experienced spine surgeon (B.v.R.) and a radiology resident (R.H.). The radiographs, MR images, and visual inspection confirmed that the lumbar spines were intact, with no fractures or slippage of the intervertebral disc present in the segments. Before mechanical testing, the spines were thawed for 18 hours in a refrigerator at $8^{\circ} \mathrm{C}$ while wrapped in saline gauzes and double plastic bags. When thawed, all muscle tissue and excessive soft tissue was removed, while all stabilizing ligaments (i.e., anterior and posterior longitudinal ligaments, interspinous and supraspinous ligaments, facet capsular ligament, and the flavum ligaments) were kept intact. To prevent dehydration, the spines were wrapped in saline-soaked gauze before testing and in between tests.

\section{Mechanical Testing}

The vertebral bodies of T12 and L5 were embedded in a casting mold using a low-melting-point $\left(48^{\circ} \mathrm{C}\right)$ bismuth alloy, with screws attached to the upper and lower vertebral bodies to optimize the fixation between the vertebrae and alloy. The spines were placed in a previously described ${ }^{4,6,7,9}$ custom-made 4-point bending device in which moments can be applied by a hydraulic materials-testing machine (model 8872, Instron) (Fig. 1). Pins mounted with 3 Optotrak (Optotrak Certus, Northern Digital Inc.) motion-capture LEDs each were attached to the anterior side of the vertebral bodies of L1-4 and to the casting molds in order to track and capture the motion pattern. The mechanical tests consisted of a set of 3 consecutive loading cycles from $-4 \mathrm{Nm}$ to $4 \mathrm{Nm}$ at an angular velocity of $0.5 \%$ second and were performed at room temperature. The moments were applied consecutively in 3 different loading directions: flexion/extension (FE), lateral bending (LB), and axial rotation (AR). To correct for order effects, the order of directions was balanced and alternated between LB-FE-AR and AR-FE-LB. Since the test setup had to be converted for each test, there were approximately $5 \mathrm{~min}$ utes between each loading direction.

\section{Surgical Procedures}

A single-level laminectomy was performed and consisted of removal of the lamina and spinous process of the apex (i.e., L3), while keeping the pars interarticularis and facet joints intact. This resulted in a disruption of the supraspinous, interspinous, and flavum ligaments. The posterior instrumentation consisted of a short instrumentation inserted between the two adjacent vertebrae to the apex (i.e., L2 and L4) with titanium rods and pedicle screws in L2 and L4, without a cross-connector. Both procedures were analogous to standard clinical practice and were performed by an experienced spine surgeon (B.v.R. or A.S.). Subsequent mechanical testing followed after both the laminectomy and posterior instrumentation.

\section{Data and Statistical Analysis}

The motion pattern of the clusters of LEDs on the 


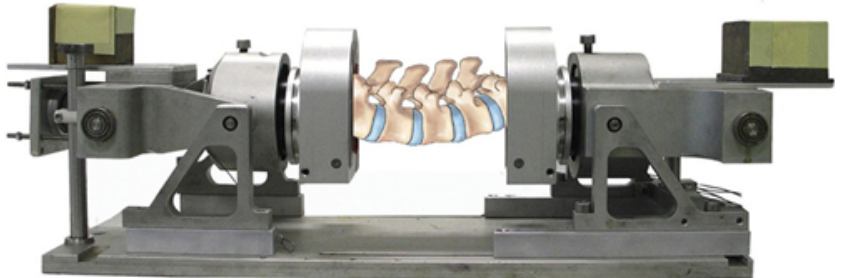

FIG. 1. The experimental test setup. The lumbar spines were placed in a custom-made, 4-point bending device.

vertebrae and casting molds was recorded by a motion analysis system (Optotrak Certus) at a sample rate of 100 samples/sec. The load-displacement data of the 4-point bending device were recorded and digitized at $100 \mathrm{~Hz}$. The ROM (in degrees) was calculated between $-4 \mathrm{Nm}$ and +4 Nm from load-displacement data for the whole spine (i.e., T12-L5) and for each motion segment (i.e., T12-L1, L1-2, L2-3, L3-4, and L4-5) using a custom MATLAB program (MathWorks) to calculate Euler angles with Euler decomposition, always starting with the angle in the loaded direction. The stiffness (in $\mathrm{Nm} /$ degree) within the NZ for the whole spine and per motion segment was calculated from the load-displacement data between -1 and $1 \mathrm{Nm} .{ }^{4,11}$ To limit viscoelastic effects, the third cycle of the 3 loading cycles per direction was used for analysis. ${ }^{28,50}$ Because most of the NZ stiffness data appeared to be not normally distributed (Shapiro-Wilk test), the effect of laminectomy and posterior instrumentation on NZ stiffness was analyzed using Friedman tests and post hoc Wilcoxon signed-rank tests. For ROM, one-way ANOVAs with repeated measures and post hoc Friedman's leastsquare difference were used to analyze the effect of the surgical procedures. ${ }^{32,40}$ Spearman's rank correlation coefficient $(\rho)$ was used to study the effect of DD on ROM and NZ stiffness. All statistical tests were performed by using IBM SPSS Statistics for Windows, Version 22.0 (IBM Corp.).

\section{Results}

One spine was excluded after testing due to severe osteoporosis, which prevented rigid attachment of the LED markers. Nine spines were left for further analysis.

\section{Full Spines}

There was a significant main effect of the surgical pro- cedures on the ROM and NZ stiffness of the full spinal segment (i.e., T12-L5) in all loading directions $(\mathrm{p}<0.05)$ (Figs. 2 and 3).

The laminectomy procedure resulted in a mean increase in the ROM of $9.5 \%$ in FE (p=0.04) and $4.6 \%$ in LB ( $\mathrm{p}=0.01)$, and a nonsignificant increase of $4.7 \%$ in AR $(p=0.18)$. For NZ stiffness, there were no significant effects of laminectomy (Figs. 2 and 3).

Posterior instrumentation resulted in a significant decrease in ROM in all loading directions, both relative to the native state $(-22.2 \%,-24.4 \%$, and $-17.6 \%$ for FE, LB, and AR, respectively; all $\mathrm{p}<0.05)$ and compared to after laminectomy $(-28.9 \%,-27.8 \%$, and $-21.3 \%$ for FE, LB, and AR, respectively; all $\mathrm{p}<0.05$ ). NZ stiffness was increased by posterior instrumentation, compared to both the native state $(+44.7 \%,+51.7 \%$, and $+35.2 \%$ for FE, $\mathrm{LB}$, and AR, respectively; all $\mathrm{p}<0.05)$ and after laminectomy $(+50.7 \%,+50.4 \%$, and $+35.0 \%$ for FE, $\mathrm{LB}$, and $\mathrm{AR}$, respectively; all $\mathrm{p}=0.01$ ).

\section{Untreated Individual Segments}

There was no effect of surgical treatment on the ROM of the nontreated segments (T12-L1, L1-2, and L4-5 [see Table 1]). Furthermore, no significant changes in NZ stiffness were observed after laminectomy in the nontreated segments. Posterior instrumentation only increased NZ stiffness in LB on level T12-L1, with $37.5 \%$ compared to native state and $14.5 \%$ compared to after laminectomy (both $\mathrm{p}<0.05$ ), and on L1-2 with $48.0 \%$ compared to the state after laminectomy $(\mathrm{p}=0.01)$ (Table 2$)$. The NZ stiffness of L4-5 was not affected by the surgical procedures.

\section{Treated Individual Segments}

Laminectomy decreased the NZ stiffness, with $22.5 \%$ on L3-4 in LB ( $p=0.04)$ (Table 2), but it did not significantly alter the ROM of the treated segments (Table 1). Posterior instrumentation, however, caused a significant decrease in the ROM of the treated segments in FE and LB relative to both the laminectomy and the native state (Table 1). AR ROM was only decreased in L2-3, with $40.4 \%$ compared to after laminectomy $(p=0.04)$. Severe increases in NZ stiffness after placement of posterior instrumentation were found, especially in FE, with $236 \%$ on L2-3 and $143.9 \%$ on L3-4, both relative to the native state (both p < 0.03), and an increase of $303.7 \%$ on L2-3 and $214.8 \%$ on L3-4 relative to the state after laminectomy

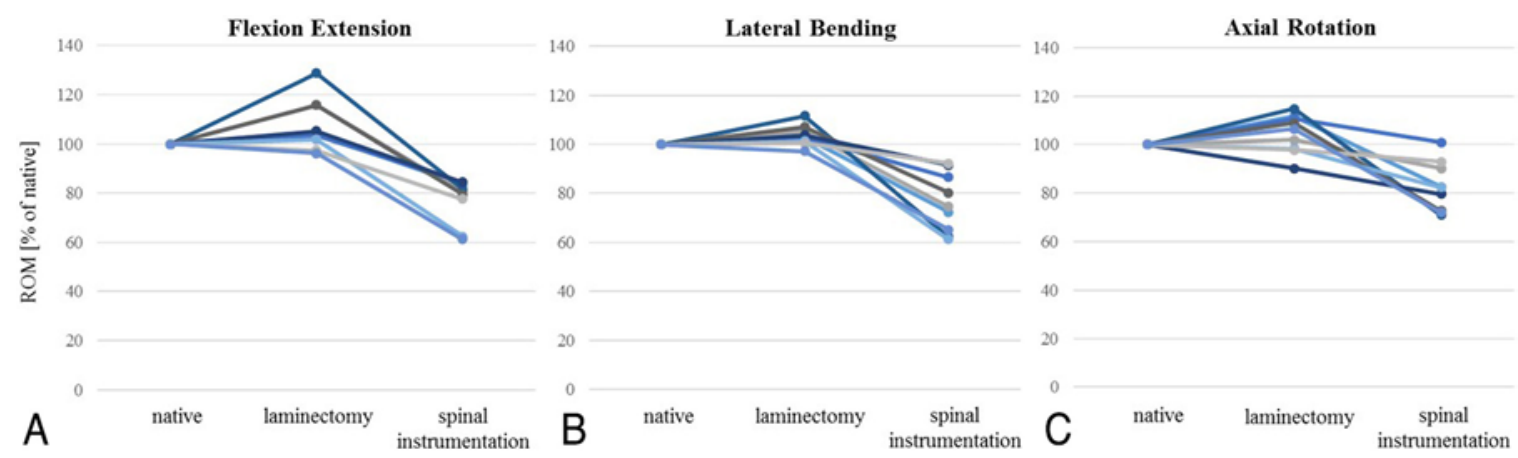

FIG. 2. The ROM per spine (T12-L5) after laminectomy and placement of posterior instrumentation, compared to native state. 


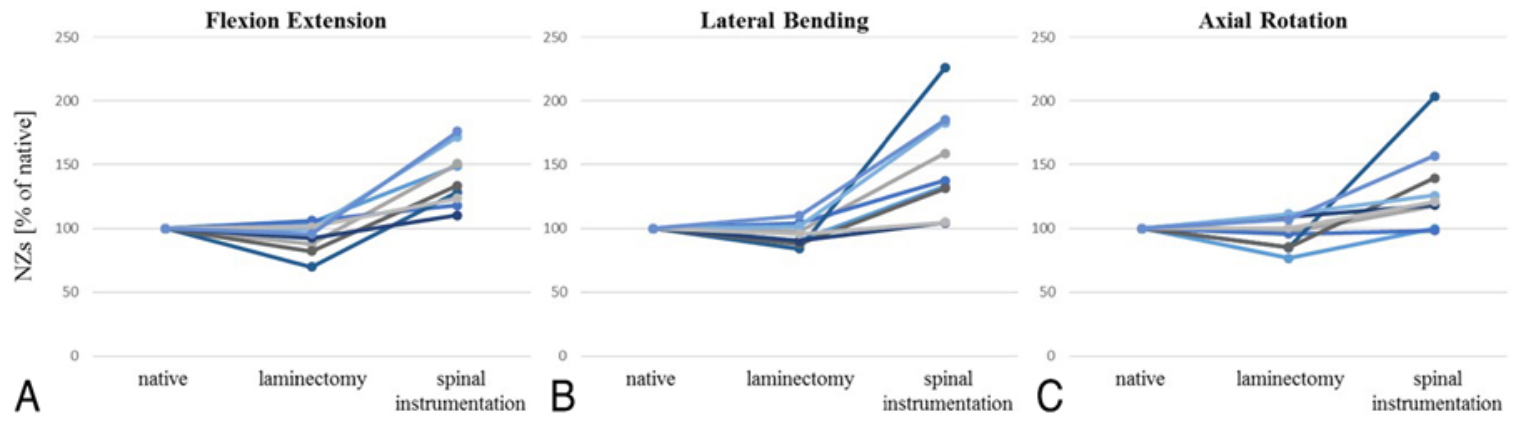

FIG. 3. The NZ stiffness per spine (T12-L5) after laminectomy and placement of posterior instrumentation, compared to native state.

(both $\mathrm{p}=0.01$ ). On L2 -3 , there was an increase in LB of $189.5 \%$ relative to the native state and $211.7 \%$ relative to the state after laminectomy (both $\mathrm{p}<0.05$ ).

\section{Correlations Between Disc Degeneration and Stability}

For intervertebral DD, there was a negative correlation with ROM and a positive correlation with NZ stiffness in FE and LB in the segments around the apex (i.e., L2-4) (Table 3). There was no effect of DD on the non-apex segments (i.e., T12-L1, L1-2, and L4-5).

\section{Discussion}

Patients suffering from de novo degenerative lumbar scoliosis are often disabled by severe neurological symptoms, caused by spinal canal stenosis. ${ }^{1}$ To relieve symptoms, single-level laminectomy followed by posterior instrumentation is a common surgical treatment. ${ }^{20}$ However, the effect of these procedures on the stability of the scoliotic lumbar spine is unknown, and thus, it is not known whether posterior instrumentation is necessary after laminectomy to prevent instability.

In this study, we found that laminectomy causes a 9.5\% increase in the ROM for the entire spine in FE and a $4.6 \%$ increase in LB, although no significant increase was found in the ROM of any individual segment. There was only a significant decrease in the stiffness of the spine in FE on L3-4, which was the lower adjacent segment of the apex of the curve. For posterior instrumentation, however, there was a strong decrease in ROM and a substantial increase in the stiffness of both the whole lumbar spine and individual, treated levels in most directions.

We also showed that intervertebral DD had a negative correlation with the ROM and a positive correlation with the NZ stiffness in FE and LB around the apex, suggesting that the degree of DD has a detrimental effect on the flexibility of the spinal segment around the apex.

The native ROM of the degenerative scoliotic spines studied here was lower than the reported ROM of nonscoliotic spines from the same population, studied using the same methodology. ${ }^{3,7}$ Conversely, the NZ stiffness was found to be higher than that reported in nonscoliotic spines. ${ }^{3,7}$ After laminectomy, the ROM and NZ stiffness values are still lower and higher than those of nonscoliotic spine, respectively. This suggests that laminectomy does not result in instability in degenerative scoliotic spines. After instrumentation, the ROM and NZ stiffness further deviate from native values found in nonscoliotic spines. ${ }^{3,7}$ An explanation for the relatively stiff spines may be the degeneration of the intervertebral discs, which were found to be positively related to a decreased ROM and increased NZ stiffness. Faraj et al. have also found that increased intervertebral DD is associated with curvature progression in cases of degenerative lumbar scoliosis, which may be a sign of progression of the disease itself.22 That severe intervertebral DD stiffens the spine has also been previously reported by several groups in nonscoliotic spines. $5,41,44$

These biomechanical results provide a fundamental support for several clinical observations. For instance, Masuda et al. found that the Cobb angle does not progress after decompression alone, which indicates that the spine remains stable after decompression. ${ }^{39}$ Kleinstück et al. compared decompression alone, limited fusion without deformity correction, and long-segment fusion with deformity correction in degenerative lumbar scoliosis and found no difference in patient-reported outcome measures after a 2-year follow-up period. ${ }^{38}$ Similarly, in randomized controlled trials of patients with lumbar spinal canal stenosis, with or without degenerative spondylolisthesis, decompressive surgery followed by the placement of posterior instrumentation did not result in better clinical outcomes after 2 and 5 years of follow-up than decompressive surgery alone. ${ }^{23,36,39}$ Notably, Daubs et al. compared the duration of the symptom-free results after decompression alone with decompression and fusion surgery in patients with mild degenerative lumbar scoliosis $\left(<30^{\circ}\right)$ and stenosis after long periods of time. At 5 years postoperatively, $12(75 \%)$ of the 16 patients in the decompression-alone group developed recurrent stenosis versus only 14 (36\%) of 39 patients in the decompression and fusion group ( $\mathrm{p}$ $=0.016) .{ }^{19}$ The results of Daubs et al. indicate that decompression alone may result in a return of stenotic symptoms (i.e., progressive instability) compared to decompression and fusion after long periods of time in the setting of mild degenerative lumbar scoliosis and symptomatic stenosis. ${ }^{19}$

The prevalence of degenerative lumbar scoliosis ranges from $1 \%$ to $10 \%$, and is reported to be more than $30 \%$ in asymptomatic elderly subjects. ${ }^{45}$ Patients with degenerative lumbar scoliosis present at a relatively high age, often with several comorbidities and poor bone quality. ${ }^{1,25,45,52}$ This increases the risk of intra- and postoperative complications, ${ }^{15,21}$ which is shown by a study of Transfeldt et al., who reported a complication rate of 40\%-56\% after spinal fusion, compared to only $10 \%$ in decompression alone. ${ }^{47}$ 

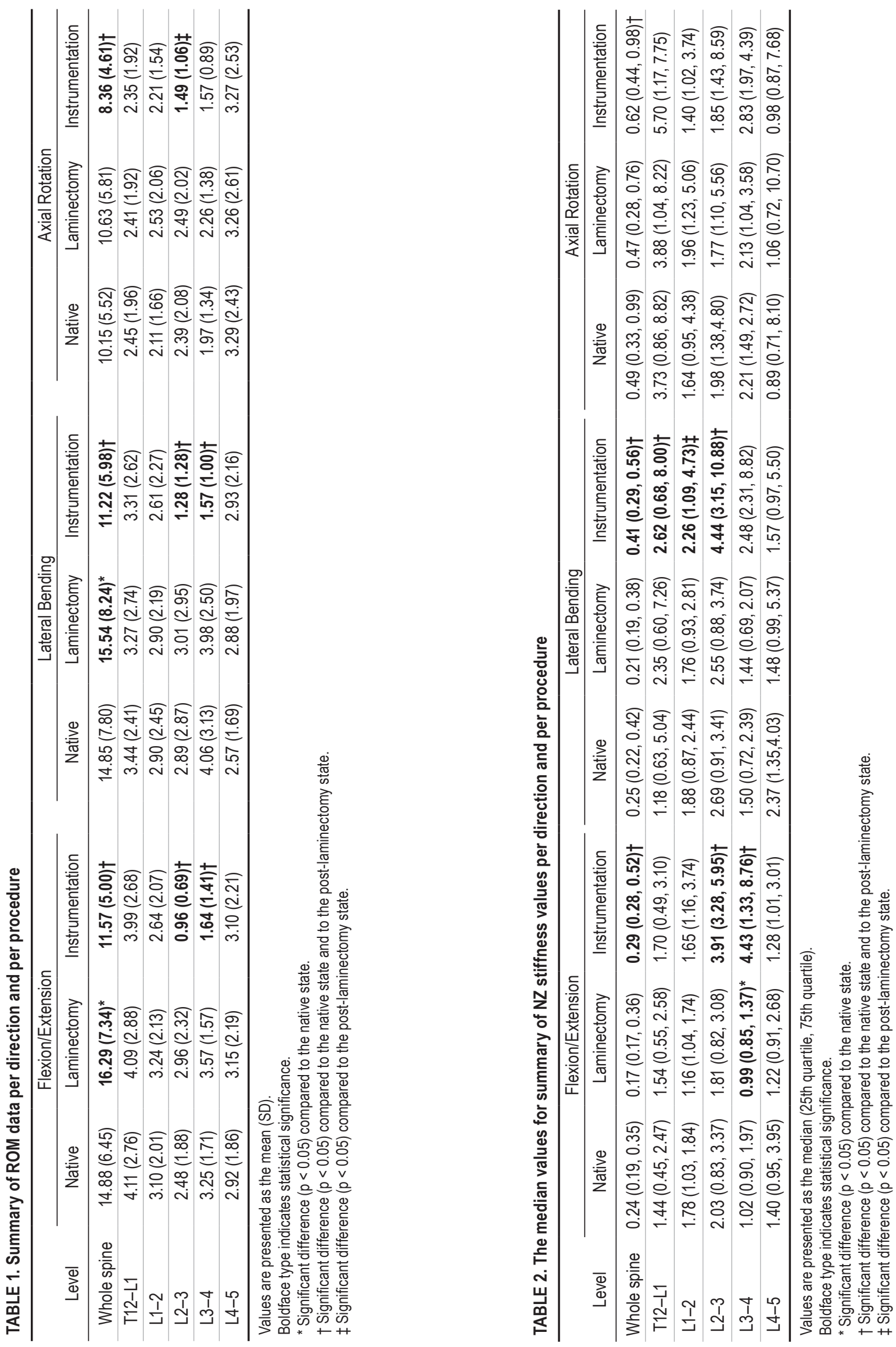
TABLE 3. Summary of Spearman rank correlation coefficient data between DD and native ROM and NZ stiffness

\begin{tabular}{|c|c|c|c|c|c|c|}
\hline \multirow[b]{2}{*}{ Level* $^{*}$} & \multicolumn{3}{|c|}{ ROM } & \multicolumn{3}{|c|}{ NZ Stiffness } \\
\hline & $\mathrm{FE}$ & LB & AR & FE & LB & AR \\
\hline All segments & -0.256 & -0.279 & -0.189 & 0.246 & 0.281 & 0.137 \\
\hline Non-apex & -0.230 & -0.334 & -0.141 & 0.198 & 0.366 & 0.050 \\
\hline Apex & -0.666 & -0.599 & -0.429 & 0.640 & 0.537 & 0.307 \\
\hline
\end{tabular}

Boldface type indicates statistical significance $(p<0.05)$.

${ }^{*}$ Apex $=\mathrm{L} 2-4$; non-apex $=\mathrm{T} 12-\mathrm{L} 2$ and $\mathrm{L} 4-5$.

Decompression of the spinal canal, achieved by performing a laminectomy, which is not followed by posterior instrumentation, may avoid the risk of intra- and postoperative complications attendant on posterior instrumentation (e.g., neurological deficit, screw malposition, proximal junctional kyphosis, or instrumentation failure). ${ }^{14,27}$

This study has several limitations. Despite inherent design weaknesses that are mostly related to the difficulty for in vitro studies to precisely replicate the biomechanical effects of decompression and instrumentation in patients with scoliotic spines, it has to be noted that all radiographic images of the specimens were taken in a supine position rather than a more natural weight-bearing standing position, which is known to increase Cobb angle measurements in patients with scoliosis and might explain why we did not find any lateral slippage. ${ }^{10,51}$ Also, during mechanical testing, we did not apply compressive axial loading. However, applying axial compression while testing would create additional moments, especially in spines with various degrees of scoliosis, that could interfere with the loading directions and consequently have an effect on the loaddisplacement data. ${ }^{37}$ While a follower load could potentially reduce buckling problems with compression, follower loads can never precisely and continuously be applied through the center of rotation during spine bending and therefore may cause substantial additional bending moments of unknown magnitude. Moreover, follower loads have been shown to cause substantial shear loads. ${ }^{18}$ The amount of loading and the loading directions are based on previous studies and represent bending moments that normally act on lumbar spines ${ }^{4,6,7,11,12,31,49,50}$ and are similar to previous work, thereby allowing quantitative comparisons. However, no failure tests were performed, as the aim of this study was to assess the stability of lumbar spines with degenerative scoliosis. The results should be interpreted with caution, as the human cadaveric model only resembles the patient model and is not identical to patients. The spines were carefully selected for scoliosis and degenerative changes such as DD and osteophytes, characteristic of the findings in patients with degenerative lumbar scoliosis. Unfortunately, the medical records were unavailable, and it is unknown whether these radiographic features represented true degenerative lumbar scoliosis or adolescent idiopathic scoliosis with subsequent spinal degeneration. However, in a general elderly population, the prevalence of degenerative lumbar scoliosis can be expected to be much higher than that of adolescent idiopathic scoliosis. ${ }^{45}$ Moreover, it should be noted that the present study did not use dual-energy x-ray absorptiometry (a.k.a., DEXA) scans to assess for osteoporosis, which is of particular interest when considering surgical treatment in elderly patients with degenerative lumbar scoliosis. ${ }^{2}$ The presence of osteoporosis does not necessarily preclude surgical treatment, but low bone quality may alter the biomechanical effect of decompression alone or decompression with instrumentation. Future studies on the effect of osteoporosis on spinal stability after these surgical procedures are therefore warranted. Also, some differences in ROM of NZs after the surgical procedures were quite large but not significant. For example, there was a $45.6 \%$ decrease in NZ stiffness at L3-4 in AR after laminectomy, with a p value of 0.07 . This suggests that the statistical power of the study is low due to the limited number of human cadaveric specimens that could be included. Due to the use of this study population, we were unable to follow these patients over time, and thus, it was impossible to study the effect of the surgical procedures over time. Since degeneration is a slowly occurring process, progressive instability might develop over time after decompression, and thus, long-term changes are clinically important as well, such as shown by the previously mentioned study by Daubs et al. ${ }^{19}$ However, several clinical studies with up to 5 years of follow-up show good clinical outcome, which suggests that the immediate effect of the surgical procedures by directly altering spinal biomechanics is greater than the slow effect of end-stage degeneration..$^{23,36,39}$ For posterior instrumentation, there were screws placed in L2 and L4, which were connected with bilateral rods. No screws were placed in L3, as this would increase the difficulty of placing the rods in the screws at this level due to the variety in Cobb angle of the lumbar spines. This pragmatic setup was chosen since our aim was to stabilize the spine after the decompression, rather than to correct the deformity. In patients, placing screws at the level of decompression is common practice. However, placing screws at the level of the apex would only increase stiffness, as has been shown by the biomechanical study of Hart et al. ${ }^{34}$ Finally, we did not take foraminal stenosis into account, which is known to be prevalent in degenerative lumbar scoliosis. ${ }^{24}$ It would be interesting to study the biomechanical effect of widening the foramen (i.e., removing the facet joint) at the concave site of curve in further research.

\section{Conclusions}

This study evaluated the short-term biomechanical effects of spine surgery on degenerative scoliotic lumbar spines of human cadavers. The ROM of lumbar spines with degenerative scoliosis is slightly increased by laminectomy, while the NZ stiffness of lumbar spines is not affected, and both remain within physiological range. How- 
ever, posterior instrumentation results in a severe decrease in ROM and increase in NZ stiffness. This indicates that the laminectomy does not result in severe instability, whereas spinal fusion results in a rigid construct. This suggests that patients with degenerative lumbar scoliosis may be served better by undergoing a laminectomy alone, as this would, in cases of symptomatic spinal stenosis due to degenerative lumbar scoliosis, relieve them from the disabling symptoms by widening the spinal canal, whereas spinal instrumentation might not be necessary, as this only causes an immediate, severe decrease in ROM and increase in NZ stiffness. Long-term effects should nevertheless be studied before implementing this approach into the clinic.

\section{References}

1. Aebi M: The adult scoliosis. Eur Spine J 14:925-948, 2005

2. Ailon T, Smith JS, Shaffrey CI, Lenke LG, Brodke D, Harrop JS, et al: Degenerative spinal deformity. Neurosurgery 77 (Suppl 4):S75-S91, 2015

3. Bisschop A, Holewijn RM, Kingma I, Stadhouder A, Vergroesen PPA, van der Veen AJ, et al: The effects of single-level instrumented lumbar laminectomy on adjacent spinal biomechanics. Global Spine J 5:39-48, 2015

4. Bisschop A, Kingma I, Bleys RLAW, Paul CPL, van der Veen AJ, van Royen BJ, et al: Effects of repetitive movement on range of motion and stiffness around the neutral orientation of the human lumbar spine. J Biomech 46:187-191, 2013

5. Bisschop A, Mullender MG, Kingma I, Jiya TU, van der Veen AJ, Roos JC, et al: The impact of bone mineral density and disc degeneration on shear strength and stiffness of the lumbar spine following laminectomy. Eur Spine J 21:530-536, 2012

6. Bisschop A, van Dieën JH, Kingma I, van der Veen AJ, Jiya TU, Mullender MG, et al: Torsion biomechanics of the spine following lumbar laminectomy: a human cadaver study. Eur Spine J 22:1785-1793, 2013

7. Bisschop A, van Engelen SJPM, Kingma I, Holewijn RM, Stadhouder A, van der Veen AJ, et al: Single level lumbar laminectomy alters segmental biomechanical behavior without affecting adjacent segments. Clin Biomech (Bristol, Avon) 29:912-917, 2014

8. Bisschop A, van Royen BJ, Mullender MG, Paul CPL, Kingma I, Jiya TU, et al: Which factors prognosticate spinal instability following lumbar laminectomy? Eur Spine J 21:2640-2648, 2012

9. Bramer JAM, Barentsen RH, vd Elst M, de Lange ESM, Patka P, Haarman HJTM: Representative assessment of long bone shaft biomechanical properties: an optimized testing method. J Biomech 31:741-745, 1998

10. Brink RC, Colo D, Schlösser TPC, Vincken KL, van Stralen $\mathrm{M}$, Hui SCN, et al: Upright, prone, and supine spinal morphology and alignment in adolescent idiopathic scoliosis. Scoliosis Spinal Disord 12:6, 2017

11. Busscher I, van der Veen AJ, van Dieën JH, Kingma I, Verkerke GJ, Veldhuizen AG: In vitro biomechanical characteristics of the spine: a comparison between human and porcine spinal segments. Spine (Phila Pa 1976) 35:E35-E42, 2010

12. Busscher I, van Dieën JH, Kingma I, van der Veen AJ, Verkerke GJ, Veldhuizen AG: Biomechanical characteristics of different regions of the human spine: an in vitro study on multilevel spinal segments. Spine (Phila Pa 1976) 34:28582864, 2009

13. Cho KJ, Kim YT, Shin SH, Suk SI: Surgical treatment of adult degenerative scoliosis. Asian Spine J 8:371-381, 2014
14. Cho KJ, Suk SI, Park SR, Kim JH, Kim SS, Choi WK, et al: Complications in posterior fusion and instrumentation for degenerative lumbar scoliosis. Spine (Phila Pa 1976) 32:2232-2237, 2007

15. Cloyd JM, Acosta FL Jr, Cloyd C, Ames CP: Effects of age on perioperative complications of extensive multilevel thoracolumbar spinal fusion surgery. J Neurosurg Spine 12:402408, 2010

16. Cobb JR: The problem of the primary curve. J Bone Joint Surg Am 42-A:1413-1425, 1960

17. Cobb JR: Scoliosis; quo vadis. J Bone Joint Surg Am 40A:507-510, 1958

18. Cripton PA, Bruehlmann SB, Orr TE, Oxland TR, Nolte LP: In vitro axial preload application during spine flexibility testing: towards reduced apparatus-related artefacts. J Biomech 33:1559-1568, 2000

19. Daubs MD, Lenke LG, Bridwell KH, Cheh G, Kim YJ, Stobbs G: Decompression alone versus decompression with limited fusion for treatment of degenerative lumbar scoliosis in the elderly patient. Evid Based Spine Care J 3:27-32, 2012

20. Epstein NE: Surgical management of lumbar stenosis: decompression and indications for fusion. Neurosurg Focus 3(2):e1, 1997

21. Faraj SSA, Haanstra TM, Martijn H, de Kleuver M, van Royen BJ: Functional outcome of non-surgical and surgical management for de novo degenerative lumbar scoliosis: a mean follow-up of 10 years. Scoliosis Spinal Disord 12:35, 2017

22. Faraj SSA, Holewijn RM, van Hooff ML, de Kleuver M, Pellisé F, Haanstra TM: De novo degenerative lumbar scoliosis: a systematic review of prognostic factors for curve progression. Eur Spine J 25:2347-2358, 2016

23. Försth P, Ólafsson G, Carlsson T, Frost A, Borgström F, Fritzell $\mathrm{P}$, et al: A randomized, controlled trial of fusion surgery for lumbar spinal stenosis. N Engl J Med 374:1413-1423, 2016

24. Fu KMG, Rhagavan P, Shaffrey CI, Chernavvsky DR, Smith JS: Prevalence, severity, and impact of foraminal and canal stenosis among adults with degenerative scoliosis. Neurosurgery 69:1181-1187, 2011

25. Fu KMG, Smith JS, Polly DW Jr, Perra JH, Sansur CA, Berven $\mathrm{SH}$, et al: Morbidity and mortality in the surgical treatment of 10,329 adults with degenerative lumbar stenosis. J Neurosurg Spine 12:443-446, 2010

26. Fu YS, Zeng BF, Xu JG: Long-term outcomes of two different decompressive techniques for lumbar spinal stenosis. Spine (Phila Pa 1976) 33:514-518, 2008

27. Ghobrial GM, Williams KA Jr, Arnold P, Fehlings M, Harrop JS: Iatrogenic neurologic deficit after lumbar spine surgery: a review. Clin Neurol Neurosurg 139:76-80, 2015

28. Goel VK, Panjabi MM, Patwardhan AG, Dooris AP, Serhan $\mathrm{H}$ : Test protocols for evaluation of spinal implants. J Bone Joint Surg Am 88 (Suppl 2):103-109, 2006

29. Grubb SA, Lipscomb HJ, Coonrad RW: Degenerative adult onset scoliosis. Spine (Phila Pa 1976) 13:241-245, 1988

30. Grubb SA, Lipscomb HJ, Suh PB: Results of surgical treatment of painful adult scoliosis. Spine (Phila Pa 1976) 19:1619-1627, 1994

31. Guan Y, Yoganandan N, Moore J, Pintar FA, Zhang J, Maiman DJ, et al: Moment-rotation responses of the human lumbosacral spinal column. J Biomech 40:1975-1980, 2007

32. Gudgeon AC: Book review: Statistical Methods for Psychology. J R Stat Soc Ser D Statistician 43:211-212, 1994

33. Guler UO, Yuksel S, Yakici S, Domingo-Sabat M, Pellise F, Pérez-Grueso FJS, et al: Analysis of the reliability of surgeons' ability to differentiate between idiopathic and degenerative spinal deformity in adults radiologically. What descriptive parameters help them decide? Eur Spine J 25:2401-2407, 2016 
34. Hart R, Hettwer W, Liu Q, Prem S: Mechanical stiffness of segmental versus nonsegmental pedicle screw constructs: the effect of cross-links. Spine (Phila Pa 1976) 31:E35-E38, 2006

35. Hosogane N, Watanabe K, Kono H, Saito M, Toyama Y, Matsumoto M: Curve progression after decompression surgery in patients with mild degenerative scoliosis. J Neurosurg Spine 18:321-326, 2013

36. Inose H, Kato T, Yuasa M, Yamada T, Maehara H, Hirai T, et al: Comparison of decompression, decompression plus fusion, and decompression plus stabilization for degenerative spondylolisthesis: a prospective, randomized study. Clin Spine Surg 31:E347-E352, 2018

37. Kingma I, Busscher I, van der Veen AJ, Verkerke GJ, Veldhuizen AG, Homminga J, et al: Coupled motions in human and porcine thoracic and lumbar spines. J Biomech 70:5158,2018

38. Kleinstück F, Dvorak J, Mannion AF: Are "structural abnormalities" on magnetic resonance imaging a contraindication to the successful conservative treatment of chronic nonspecific low back pain? Spine (Phila Pa 1976) 31:2250-2257, 2006

39. Masuda K, Higashi T, Yamada K, Sekiya T, Saito T: The surgical outcome of decompression alone versus decompression with limited fusion for degenerative lumbar scoliosis. J Neurosurg Spine 29:259-264, 2018

40. Meier U: A note on the power of Fisher's least significant difference procedure. Pharm Stat 5:253-263, 2006

41. Muriuki MG, Havey RM, Voronov LI, Carandang G, Zindrick MR, Lorenz MA, et al: Effects of motion segment level, Pfirrmann intervertebral disc degeneration grade and gender on lumbar spine kinematics. J Orthop Res 34:13891398,2016

42. Pfirrmann CWA, Metzdorf A, Zanetti M, Hodler J, Boos N: Magnetic resonance classification of lumbar intervertebral disc degeneration. Spine (Phila Pa 1976) 26:1873-1878, 2001

43. Phan K, Xu J, Maharaj MM, Li J, Kim JS, Di Capua J, et al: Outcomes of short fusion versus long fusion for adult degenerative scoliosis: a systematic review and meta-analysis. Orthop Surg 9:342-349, 2017

44. Phillips FM, Slosar PJ, Youssef JA, Andersson G, Papatheofanis F: Lumbar spine fusion for chronic low back pain due to degenerative disc disease: a systematic review. Spine (Phila Pa 1976) 38:E409-E422, 2013

45. Silva FE, Lenke LG: Adult degenerative scoliosis: evaluation and management. Neurosurg Focus 28(3):E1, 2010

46. Silvers HR, Lewis PJ, Asch HL: Decompressive lumbar laminectomy for spinal stenosis. J Neurosurg 78:695-701, 1993
47. Transfeldt EE, Topp R, Mehbod AA, Winter RB: Surgical outcomes of decompression, decompression with limited fusion, and decompression with full curve fusion for degenerative scoliosis with radiculopathy. Spine (Phila Pa 1976) 35:1872-1875, 2010

48. van Engelen SJPM, Bisschop A, Smit TH, van Royen BJ, van Dieën JH: The effect of neighboring segments on the measurement of segmental stiffness in the intact lumbar spine. Spine J 15:1302-1309, 2015

49. Wilke HJ, Jungkunz B, Wenger K, Claes LE: Spinal segment range of motion as a function of in vitro test conditions: effects of exposure period, accumulated cycles, angular-deformation rate, and moisture condition. Anat Rec 251:15-19, 1998

50. Wilke HJ, Wenger K, Claes L: Testing criteria for spinal implants: recommendations for the standardization of in vitro stability testing of spinal implants. Eur Spine J 7:148-154, 1998

51. Yang C, Li Y, Zhao Y, Zhu X, Li M, Liu G: Adult degenerative scoliosis: can Cobb angle on a supine posteroanterior radiograph be used to predict the Cobb angle in a standing position? Medicine (Baltimore) 95:e2732, 2016

52. York PJ, Kim HJ: Degenerative scoliosis. Curr Rev Musculoskelet Med 10:547-558, 2017

\section{Disclosures}

The authors report no conflict of interest concerning the materials or methods used in this study or the findings specified in this paper.

\section{Author Contributions}

Conception and design: Rustenburg, Faraj, Holewijn, Emanuel. Acquisition of data: Rustenburg, van Royen, Stadhouder, Emanuel. Analysis and interpretation of data: Rustenburg, Faraj, Holewijn, Kingma, Emanuel. Drafting the article: Rustenburg, Faraj. Critically revising the article: all authors. Reviewed submitted version of manuscript: all authors. Approved the final version of the manuscript on behalf of all authors: Rustenburg. Statistical analysis: Rustenburg, Emanuel. Study supervision: Kingma, van Royen, Stadhouder, Emanuel.

\section{Correspondence}

Christine M. E. Rustenburg: Amsterdam UMC, Amsterdam, The Netherlands.c.rustenburg@amsterdamumc.nl. 